\title{
UHE Cosmic Rays and AGN Jets
}

\section{Frank M. Rieger*}

ZAH, Institute of Theoretical Astrophysics, University of Heidelberg

Philosophenweg 12, 69120 Heidelberg, Germany

E-mail: f.riegereuni-heidelberg.de

Active Galactic Nuclei (AGN) and their relativistic jets are believed to be potential sites of ultrahigh-energy (UHE) cosmic ray acceleration. This paper reviews basic observational findings as well as requirements on source energetics, and then discusses the relevance of different acceleration sites and mechanisms, such as black hole gap, shock in back-flows or jet shear acceleration. When put in context, the result suggests that Fermi-type particle acceleration at trans-relativistic shocks and/or in shearing, relativistic flows offers the most promising framework for UHECR production in AGN. Truly deciphering the astrophysical sources of UHECRs, however, still needs improved statistical information on arrival directions and source correlations.

High Energy Phenomena in Relativistic Outflows VII - HEPRO VII

9-12 July 2019

Facultat de Fisica, Universitat de Barcelona, Spain

${ }^{*}$ Speaker. 


\section{Introduction}

The origin of the ultra-high-energy cosmic rays (UHECRs, $E>10^{18} \mathrm{eV}=1 \mathrm{EeV}$ ) is still not resolved. While considered extragalactic in origin [1], the astrophysical sources are unknown. Possible candidates include Active Galactic Nuclei (AGN) and their relativistic jets, starburst galaxies, gamma-ray bursts as well as cosmic (supercluster) shocks. The present paper focuses on the relevance of the former, and highlights some of the recent developments in the field.

Experimentally, major progress has been achieved over the last couple of years by the Pierre Auger (PA) and the Telescope Array (TA) collaborations, see e.g. refs. [2, 3] for recent reports. Both collaborations run hybrid instruments that are fully operational since 2008. The larger PA observatory consists of 1660 surface and four fluorescence detectors, and is located in the southern hemisphere (Mendoza, Argentina). The TA, on the other hand, consists of 507 surface and three fluorescence detectors, and is located in the northern hemisphere (Utah, USA). The surface detectors measure air shower particles on the ground, and are sensitive to its electromagnetic, muonic and hadronic components, while the fluorescence detectors observe the longitudinal development of air showers in the atmosphere by the light emitted during their passage.

In the following, basic experimental findings are briefly summarized. Helpful overviews of recent results and progress can also be found in refs. [4, 5].

\section{Basic Experimental Results}

\subsection{Energy Spectrum}

A decent agreement between the TA and PA experiments is found up to $E \sim 4 \times 10^{19} \mathrm{eV}$, once the data are adjusted for the uncertainty (10\%) in absolute energy scale, see e.g. ref. [6] and Fig. 1. The resultant cosmic-ray (CR) energy spectrum also reveals a pronounced steepening above $E \sim 5 \times 10^{19} \mathrm{eV}$, that is compatible with a Greisen-Zatsepin-Kuzmin (GZK) cut-off. Differences between experiments are seen, however, towards higher energies. It seems possible that these may be caused by different instrumental systematics, and/or some true source difference (as the particle mean free path increases, differences in the large-scale structure may no longer be negligible). The
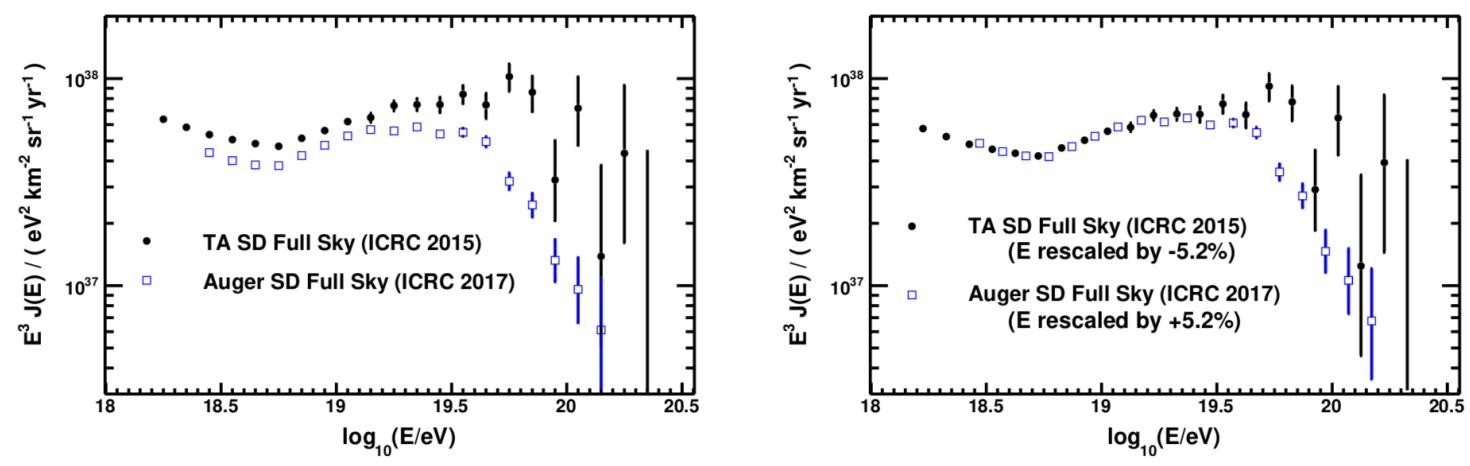

Figure 1: Energy spectrum as measured by the PA and TA surface detectors (left), adjusted for uncertainties in absolute energy scale (right). From ref. [6]. 
observed spectrum implies a UHECR luminosity density (around $10^{19.5} \mathrm{eV}$ ) of $l_{\mathrm{UHECR}} \sim 6 \times 10^{43}$ $\mathrm{erg} /\left(\mathrm{Mpc}^{3} \mathrm{yr}\right)[7]$.

\subsection{Composition}

Cosmic-ray particles entering the atmosphere induce hadronic showers by interacting with atmospheric nuclei. The atmospheric depth of the shower maximum, $X_{\max }\left[\mathrm{g} / \mathrm{cm}^{2}\right]$, is sensitive to the primary mass. In general, light particles penetrate deeper in the atmosphere and exhibit a steeper lateral distribution compared to heavy nuclei. Comparison of TA and PA data suggests that the composition becomes lighter between $10^{17.2} \mathrm{eV}$ and $10^{18.3} \mathrm{eV}$, compatible with a transition from galactic to extragalactic cosmic rays. Above $E \sim 10^{18.3} \mathrm{eV}$ the composition seems to become heavier again, as evident both, from the mean $X_{\max }$ and its fluctuations $\sigma\left(X_{\max }\right)$ measurements (see Fig. 2), with a trend that protons are gradually replaced by helium, helium by nitrogen etc, an iron contribution possibly emerging above $10^{19.4} \mathrm{eV}$, cf. ref. [8] for details.
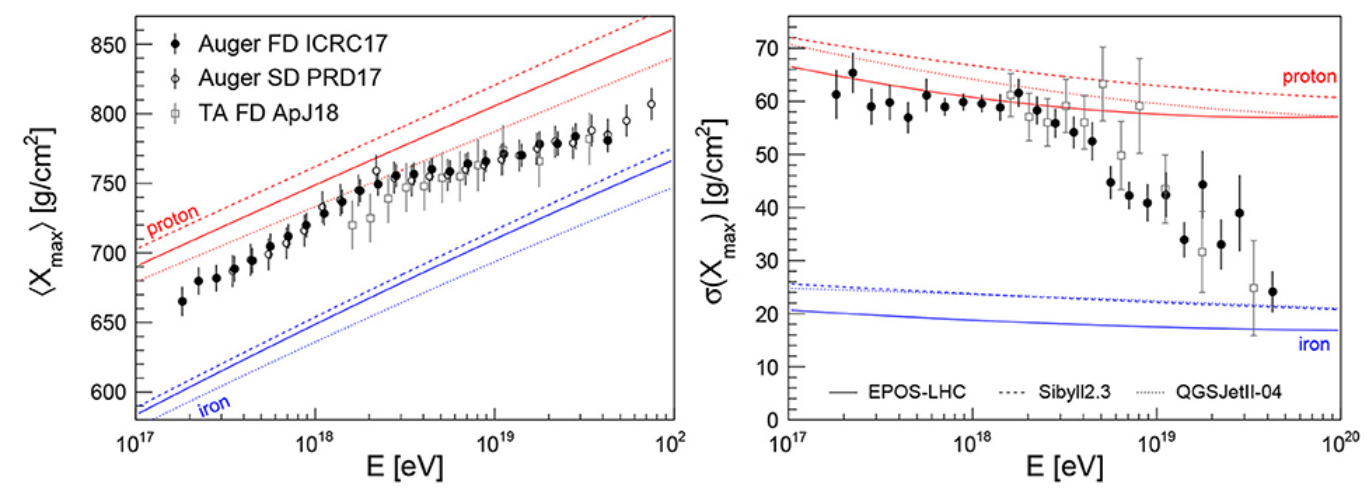

Figure 2: Composition analysis based on the mean (left) and the standard deviation (right) of the distribution of shower maximum as function of energy. The TA data have been corrected for detector effects and energy uncertainty. From ref. [8].

\subsection{Anisotropy on Intermediate Scale}

A possible anisotropy in the arrival directions of UHECRs can offer important clues as to their astrophysical origin. A recent TA analysis [9] of the anisotropy on intermediate angular scales using TA and PA events above $5.7 \times 10^{19} \mathrm{eV}$ (smeared out on circles of $25^{\circ}$ ) provides evidence for a TA hot spot (at R.A. $\sim 144^{\circ}, \operatorname{dec} \sim 40^{\circ}$ ) at a level of $5.2 \sigma$ (local significance) and $3.4 \sigma$ (global significance), respectively (cf. also [10]). This TA hot spot lies approximately in the direction of the Ursa Major/Virgo supercluster $(d \sim 18 \mathrm{Mpc})$. The PA data, on the other hand, also suggests the existence of a PA "warm" spot (local significance 3.6 $\sigma$ ), that coincides with the direction to Cen A (and the Centaurus supercluster at $d \sim 50 \mathrm{Mpc}$ ), see Fig. 3. Neither the TA nor the PA data shows any sign of excess in the direction of Virgo.

Note that if the UHECR composition would indeed become heavier $(Z \geq 10)$, as suggested in Sec. 2.2, the standard requirements on astrophysical accelerators become less dramatic. On the other hand, since particle deflection in a regular magnetic fields scales with $\theta \sim d / r_{g} \propto(Z / E)$, one 


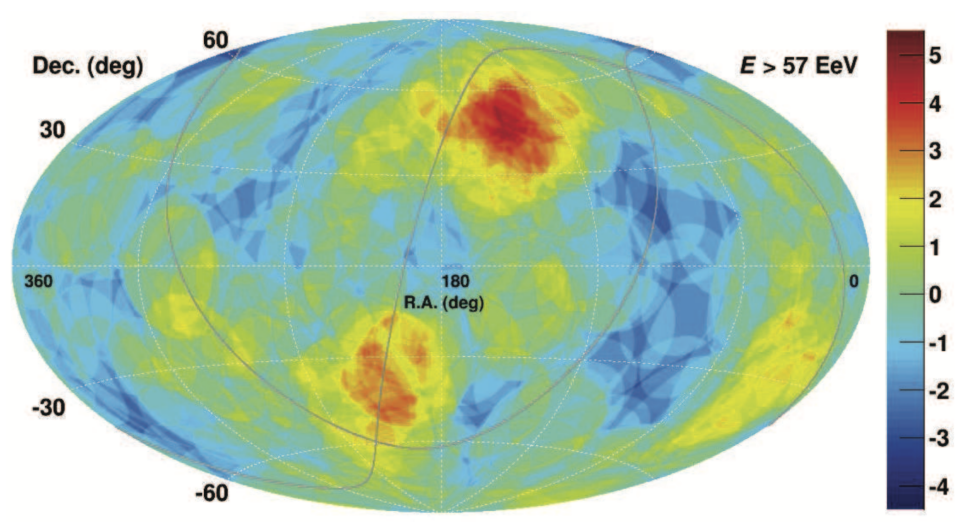

Figure 3: Sky map (Hammer-Aitoff projection in equatorial coordinates) combining TA (109 events) and PA (157 events) data with energy above $5.7 \times 10^{19} \mathrm{eV}$. The data have oversampling with a $20^{\circ}$ radius circle. No energy corrections was applied. The thin gray line above and left of the TA (upper) "hot" spot is the supergalactic plane. The PA data also suggest a (lower) "warm" spot. From ref. [9].

would then need to address why we do not observe a strong anisotropy associated with protons at $E / Z[11,12,13]$.

\subsection{Correlations with Known Sources}

The current PA analysis of the arrival direction of UHECRs above $20 \mathrm{EeV}(\sim 5500$ events) appears to favour a correlation with starburst galaxies [14]: In particular, a starburst model that attributes $9.7 \%$ of UHECRs $(>39 \mathrm{EeV})$ to nearby starburst galaxies ( 23 objects, including NGC 4945, NGC 253, M83, NGC 1068), and the remaining to isotropic background, is favoured at $4 \sigma$ over the hypothesis of isotropy, cf. also ref. [15] for update, but see also ref. [16] for a (negative) TA test. In comparison, a model of nearby $\gamma$-bright AGN (17 objects, including Cen A, M87, Mkn 421, Mkn 501, but not Fornax A), which attributes $\sim 7 \%$ of the total flux ( $>60 \mathrm{EeV}$ ) to them, is (only) favoured against isotropy at $\sim 2.7 \sigma$. It has been suggested, however, that an incorporation of Fornax A (at a distance $\sim 20 \mathrm{Mpc}$ ) could change this picture in favour of radio galaxies once magnetic deflection is properly accounted for $[17,18]$. The analysis is certainly further complicated by the fact that the CR luminosity of individual AGN is not known. In the noted PA analysis [14], the (Fermi-LAT) integral gamma-ray fluxes from $50 \mathrm{GeV}$ to $2 \mathrm{TeV}$ have been used as proxy for the UHECR flux. In the AGN case, the observable UHECR flux is then dominated (75\%) by the core of Cen A. The limitations introduced by the chosen approach necessitate further studies, part of which should include a basic treatment of magnetic deflection and the introduction of better flux proxies. As things are, it is still too early to conclude about the astrophysical sources of UHECRs.

In general, since UHECRs in AGN are confined to magnetic fields, they may escape only slowly from their sources. Since it seems likely that, e.g., Cen A and Fornax A have seen more powerful jets in the past, capable of accelerating UHECRs (see below), UHECR particles could still be escaping from their giant lobes. If this is the case, then their past activity aka source history becomes relevant. For some exemplary, recent discussion of individual source associations the reader is referred to refs. [19] (Cen A), [20] (Virgo A/M87), [21] (Cen B) and [17] (Fornax A), 
respectively.

\section{Physics Constraints}

In order for a source to be capable of accelerating UHECRs, the relevant particles need to be confined within it. This introduces a general (Hillas) bound

$$
E \leq 10^{20} Z(B / 1 \mu \mathrm{G})(L / 100 \mathrm{kpc}) \mathrm{eV},
$$

given by the condition that the particle gyro-radius, $r_{\text {gyro }}$, remains smaller than the characteristic dimension $L$ of the source. Similarly, assuming acceleration to be constrained by the motional electric field $\vec{\varepsilon}=-(\vec{V} / c) \times \vec{B}$, one obtains

$$
E \leq Z e \varepsilon L=10^{20} Z \beta(B / 1 \mu \mathrm{G})(L / 100 \mathrm{kpc}) \mathrm{eV},
$$

with $\beta=V / c$. Accordingly, UHECR acceleration generally requires fast speeds, strong magnetic fields or large source volumes [22]. The requirements are significantly relaxed if the composition at the highest energies would be heavy $(Z \geq 10)$. The above expressions, however, only provides a necessary (and not itself sufficient) condition for UHECR acceleration, and also neglect possible relativistic effects. Generalisations and improved constraints have been obtained by various considerations, e.g. $[23,24,25,11]$. In any case, the characteristic timescale for acceleration $t_{\text {acc }}$ has to be smaller than the escape $\left(\tau_{\text {esc }}\right)$ and the radiative $\left(\tau_{\text {loss }}\right)$ loss timescale, respectively. Note that since $t_{\text {acc }}$ depends on acceleration physics, and $\tau_{\text {esc }}, \tau_{\text {loss }}$ on individual source physics, this also implies that detailed ("multi-messenger") source studies become particularly interesting.

Considering shock-type particle acceleration in a relativistic outflow (of speed $\beta=V / c$ and Lorentz factor $\Gamma$ ), a generalized constraint [11] is obtained by requiring that in the local, co-moving frame $t_{\mathrm{acc}}^{\prime}<t_{\mathrm{dyn}}^{\prime}$ (with primed quantities referring to the co-moving frame). Expressing the acceleration timescale in terms of the gyro-time, i.e. $t_{\mathrm{acc}}^{\prime}=\eta t_{\mathrm{gyro}}^{\prime}=\eta E^{\prime} /\left(Z e B^{\prime} c\right), \eta \geq 1$, and the dynamical timescale as $t_{\mathrm{dyn}}^{\prime}=d /(\Gamma V)$ (with longitudinal length scale $d$ ), the maximum UHECR energy becomes

$$
E=\Gamma E^{\prime} \leq Z e B^{\prime} d /(\beta \eta) .
$$

To allow for this, the magnetic luminosity of the source, $L_{B}=2 \pi r^{2} \Gamma^{2} u_{B}^{\prime} V$ ( $r$ the lateral half width, $u_{B}^{\prime} \equiv B^{\prime 2} / 8 \pi$ ), has to be high enough. With $B^{\prime}$ from eq. (3.3) and $r \sim \theta_{j} d$ ( $\theta_{j}$ the jet half opening angle), one obtains $L_{B} \geq \theta_{j}^{2} \Gamma^{2} \eta^{2} E^{2} \beta^{3} c /(2 Z e)^{2}$, or

$$
L_{B} \gtrsim 8 \times 10^{44} \theta_{j}^{2} \Gamma^{2} \eta^{2} \beta^{3}\left(\frac{E / Z}{10^{20} \mathrm{eV}}\right)^{2} \mathrm{erg} / \mathrm{sec} .
$$

For the commonly assumed $\theta_{j} \sim 1 / \Gamma$, this thus implies a lower limit $(\eta=1)$ on the required source luminosity for steady relativistic $(\beta \sim 1)$ outflows of $L_{m} \gtrsim 8 \times 10^{44}\left([E / Z] / 10^{20} \mathrm{eV}\right)^{2} \mathrm{erg} / \mathrm{sec}$. Note that for non-relativistic shocks, $\eta \sim\left(c / V_{s}\right)^{2}$, so that $L_{m} \propto\left(c / V_{s}\right)$. The situation is again much relaxed if a heavier composition prevails at the highest end. Note that while AGN can have fast jets with maximum power reaching up to $L_{j} \sim 10^{48} \dot{m}\left(M_{B H} / 10^{9} M_{\odot}\right) \mathrm{erg} / \mathrm{sec}$ [26], the winds in starburst galaxies usually only have $L_{S B G} \sim 10^{42} \mathrm{erg} / \mathrm{sec}$ (along with low shock velocities $\sim 1000$ $\mathrm{km} / \mathrm{s}$ ) [27, 17]. Taken as face value, this would disfavour starburst galaxies as promising UHECR accelerators. 


\section{UHECR Acceleration in AGN}

UHECR acceleration in AGN may occur at various locations, close to the black hole, within their jets, at hot spot shocks or in their large-scale lobes. In the following, three recent examples will be briefly discussed.

\subsection{Black Hole Vicinity}

UHECR acceleration in the magnetospheres of rotating supermassive black holes has been considered in a number of papers, see e.g. refs. [28, 29, 30, 31, 32]. The acceleration relies on the occurrence of an electric field component parallel to the magnetic field in a charge-deficient ("gap") region close to the black hole, see also ref. [33] for a review. This could happen either at the so-called null surface (across which the Goldreich-Julian charge density, required to screen the field, changes sign) or at the stagnation surface (separating MHD in- and outflows), see Fig. 4.

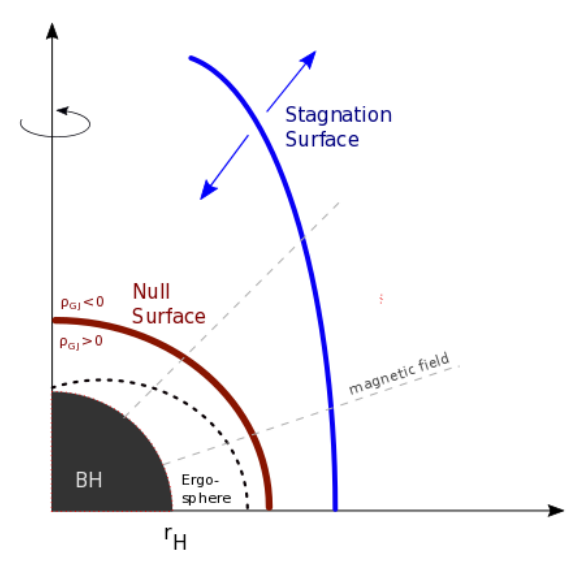

Figure 4: Illustration of the possible locations of charge-deficient regions (gaps) in rotating black hole magnetospheres where efficient UHECR acceleration may occur. The red line denotes the null surface across which the required Goldreich-Julian charge density changes sign, while the blue line delineates the stagnation surface from which stationary MHD flows start.

The maximum available voltage drop for a gap of width $h$ is of the order of [26]

$$
\Delta \Phi=\frac{1}{c} \Omega_{F} r_{H}^{2} B_{H}\left(\frac{h}{r_{g}}\right)^{2} \simeq 2 \times 10^{21} \dot{m}^{1 / 2} M_{9}^{1 / 2}\left(\frac{h}{r_{g}}\right)^{2},
$$

assuming a magnetic field $B_{H} \simeq 2 \times 10^{5} \dot{m}^{1 / 2} M_{9}^{-1 / 2} \mathrm{G}$, with $M_{9}=M_{\mathrm{BH}} / 10^{9} M_{\odot}$, field line rotation $\Omega_{F}=\Omega_{H} / 2=c / 4 r_{g}$ and gravitational radius $r_{g}=G M / c^{2}$. This would seem to suggest that in massive $\left(M_{9} \gtrsim 1\right)$, active $\left(\dot{m} \gtrsim 10^{-3}\right)$ sources, ultra-high energies $Z e \Delta \Phi \sim 10^{20} \mathrm{ZeV}$ might well be achievable.

Under realistic conditions, however, energy losses due to curvature radiation are likely to introduce 
an upper limit [26]

$$
\gamma_{\max } \simeq 100^{10} \frac{\dot{m}^{1 / 8}}{Z^{1 / 4}} M_{9}^{3 / 8}\left(\frac{h}{r_{g}}\right)^{1 / 4}
$$

for curvature radii of the order of the gravitational one. Note that this would imply a maximum energy $E_{\max }$ which no longer scales linearly with $Z$. Moreover, unless the accretion rate is low enough (usually, $\dot{m} \leq 10^{-4}$ ), the ambient soft photon environment will facilitate efficient pair production in the gap, leading to gap sizes $h \ll r_{g}$, significantly reducing achievable particle energies (eq. [4.1]). On the other hand, if accretion rates are too low (as in e.g. quiescent quasars), the expected magnetic field strength $\left(B_{H} \propto \dot{m}^{1 / 2}\right)$ would be low as well.

When taken together, this would seem to make an efficient gap-type acceleration of protons to energies much beyond $E \sim 10^{18} \mathrm{eV}$ problematic. We note that since efficient UHECR production in the black hole magnetosphere is accompanied by curvature VHE emission, gamma-ray observations may in principle allow a useful probe of UHECR acceleration, e.g. [28, 34].

\subsection{Shear in large-scale Jets}

The jets in AGN are likely to exhibit some internal jet stratification and velocity shearing, that could be conducive to efficient cosmic-ray acceleration, see ref. [35] for review and discussion. Prominent scenarios include Fermi-type particle acceleration in non-gradual (discontinuous), e.g. refs. [36, 37, 38, 39], or gradual (continuous), e.g. refs. [40, 41, 42, 43, 44], velocity shear flows.

The former utilises that if the CR particle distribution would remain nearly isotropic near a strong shear discontinuity, the mean fractional energy change for crossing and re-crossing (full cycle) is given by $\langle\Delta E / E\rangle \simeq \Gamma^{2} \beta^{2}$ [40]. This suggests that the increase in particle energy could be substantial for a velocity shear that is highly relativistic $(\Gamma \gg 1$, e.g. [38]), while for non-relativistic speeds $(\Gamma \sim 1)$ only the usual gain $\propto \beta^{2}$ is obtained. For relativistic flow speeds $(\beta \simeq 1)$, however, the non-negligible anisotropy of the particle distribution has to be modelled and taken into account. For repeated crossings, the principal effects of this is a reduction in efficiency, e.g. [36, 37].

Denoting by $\tau$ the mean cycle time (into and out of the shear), the mean acceleration timescale might in general be expressed as $t_{\mathrm{acc}} \simeq \tau /\langle\Delta E / E\rangle$. Note that, depending on the considered turbulence properties, $\tau$ might actually be dominated by the (diffusion) time a CR particle needs to return to the jet shear [39]. A related application to the kiloparsec-scale jets of FanaroffRiley (FR) I sources has been recently presented [39], see Fig. 5. The model assumes entrainment and re-acceleration of Galactic cosmic rays in a mildly relativistic shear flow ( $\beta=0.7$ ). The performed Monte Carlo simulations suggest that the escaping CRs can have quite hard spectra $\left(d N / d E \propto E^{-a}, a \lesssim 1\right)$. Furthermore, a rather complex chemical compositions at UHECR is achieved due to different injection at TeV-PeV energies $\left(E_{\text {inj,i }}=15 Z_{i} \mathrm{TeV}\right)$. This also allows to accommodate the anisotropy constraints mentioned earlier (Sec. 2.3). The maximum energy $\propto Z$ is limited by the jet size $\left(t_{\mathrm{acc}} \propto r_{j} / c\right)$ and (via $\left.\tau\right)$ dominated by the diffusion properties in the cocoon. The results shown are sensitive to the chosen cocoon properties (i.e., cocoon size, turbulence scale) and dependent on a thin velocity transition layer $\Delta r \sim r_{j} / 100$ (defining the required energy of the injected galactic cosmic rays). Enlarging $\Delta r$, for example, is likely to affect the outcome. Nevertheless, these simulations show that non-gradual shear acceleration in large-scale jets of FR I (and not only FR II) could in principle play an important role in UHECR acceleration. Given an FR I num- 

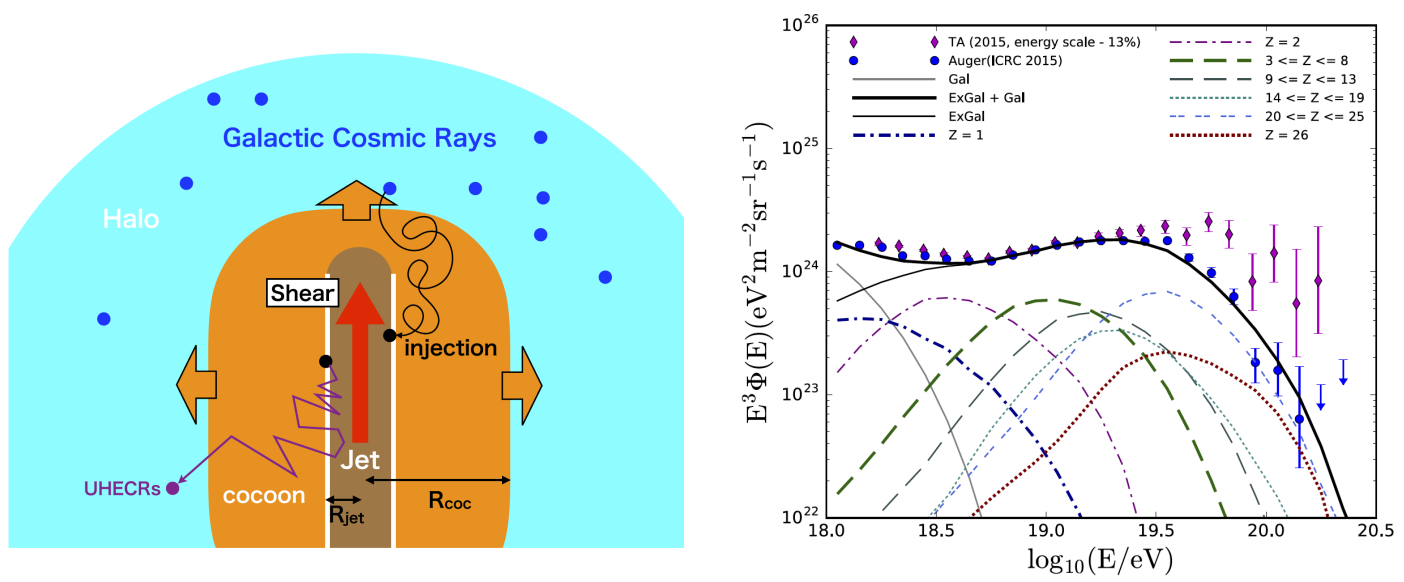

Figure 5: Left: Sketch of the considered FR I model assuming a recycling of galactic cosmic rays by nongradual shear particle acceleration in a jet - (turbulent) cocoon system. Some fraction of galactic cosmic rays is considered to be swept up ("injected") and reaccelerated to ultra-high energies. The return probability (and thus the cycle time) of a particle is dominated by the scattering (turbulence) properties in the cocoon. Right: Reconstruction of the observed UHECR spectrum assuming a mildly relativistic $(\beta \simeq 0.7)$ jet of width $r_{j}=0.5 \mathrm{kpc}$ surrounded by a thin velocity transition layer $\Delta r=5 \mathrm{pc}\left(B_{j}=0.3 \mathrm{mG}\right)$. The chemical composition at the highest energies is dominated by intermediate and heavy nuclei. From ref. [39].

ber density of $n_{F R I} \sim 10^{-5}-10^{-4} \mathrm{Mpc}^{-3}$, an average source luminosity $L \sim 2 \times 10^{40}-2 \times 10^{41}$ $\mathrm{erg} / \mathrm{sec}$ would be needed, cf. Sec. 2.1 .

If the transition layer becomes larger, particle scattering will occur within the shear layer, facilitating gradual shear particle acceleration within it, see e.g. refs. [40, 41, 42, 43, 44]. The underlying mechanism can be viewed as a stochastic, second-order Fermi-type particle acceleration process, where the usual scattering center speed is replaced by an effective velocity $\bar{u}$ determined by the shear flow profile, e.g., $\bar{u}=\left(\partial u_{z} / \partial r\right) \lambda$ in the case of a simple continuous (non-relativistic) velocity shear $\vec{u}=u_{z}(r) \vec{e}_{z}$, cf. ref. [35] for a recent review. Accordingly, the fractional energy change scales as

$$
\langle\Delta E / E\rangle \propto\left(\frac{\bar{u}}{c}\right)^{2} \propto\left(\frac{\partial u_{z}}{\partial r}\right)^{2} \lambda^{2} .
$$

This suggests a scaling for the characteristic acceleration timescale of $t_{\mathrm{acc}} \simeq \tau /\langle\Delta E / E\rangle \propto 1 / \lambda$, which, in contrast to classical first-order Fermi (shock) as well as non-gradual shear acceleration, is inversely proportional to the particle mean free path $\lambda=c \tau$. This seemingly unusual behaviour relates to the fact that as a particle increases its energy $(E \simeq p c)$, and thereby its mean free path (typically, $\lambda(p) \propto p^{\alpha}, \alpha>0$ ), a higher effective velocity $\bar{u}$ is experienced.

Gradual shear particle acceleration is particularly interesting as it could offer an explanation for the origin of the extended synchrotron X-ray emission in large-scale AGN jets that requires the maintenance of ultra-relativistic electrons $\left(\gamma_{e} \sim 10^{8}\right)$ on kpc-scales [42]. Efficient operation generally requires sufficiently relativistic flow speeds, i.e. fast jets (bulk Lorentz factors of some few) or a strong jet-back-flow system [43, 44, 35].

To assess its potential for UHECR acceleration, cf. Sec. 3, the properties of a source need to be 
such as to allow (i) CRs to be confined within its jet, and to enable CR acceleration (ii) to proceed faster than radiative losses $\left(t_{\mathrm{acc}}<t_{\mathrm{syn}}\right)$ and (iii) to operate within the lifetime $\left(t_{\mathrm{dyn}}\right)$ of the system. A related application following ref. [42] is shown in Fig. 6 assuming a linearly decreasing flow profile with $\Gamma=2$ on the jet axis. A jet-width to length ratio $\Delta r / d=0.02$ has been employed. These

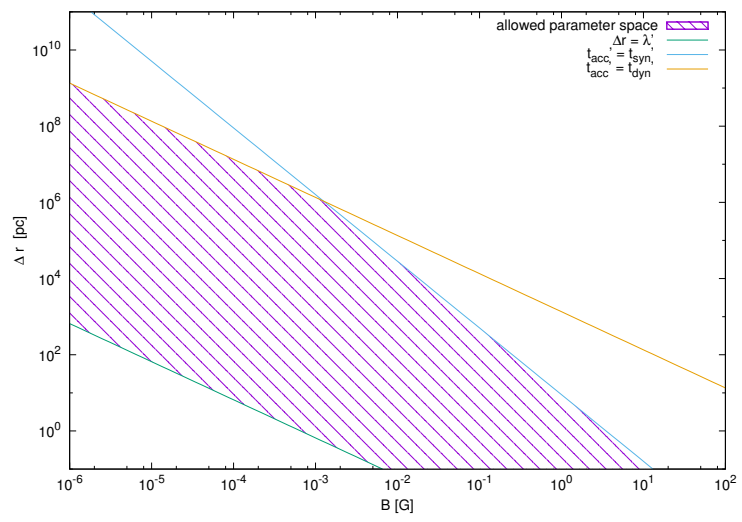

Figure 6: Permitted (hatched) parameter range (magnetic field strength $B$, shear layer width $\Delta r$ ) to allow gradual shear acceleration of protons to $\sim 10^{18} \mathrm{eV}$ and to satisfy confinement and synchrotron loss constraints. A Kolmogorov-type scaling for the particle mean free path $(q=2-\alpha=5 / 3)$ has been assumed. The required conditions for UHE proton acceleration might be met in the large-scale jets of AGN.

results suggest that proton energies $\sim 10^{18} \mathrm{eV}$ are in principle achievable for relatively plausible parameters (e.g., jet lengths $10 \mathrm{kpc}-1 \mathrm{Mpc}$, magnetic fields $B \sim[1-100] \mu \mathrm{G}$ ). Higher energies might be obtained for faster flows or heavier particles [42, 43, 44].

\subsection{Multiple shocks in back-flows}

Following the considerations in Sec. 3 and eq. (3.2), high speeds are seemingly conducive for efficient UHECR acceleration. While highly relativistic shocks might thus appear most promising, closer studies however reveal them to be problematic instead, see e.g. refs [45, 46, 47]. This is partly related to the fact that highly relativistic shocks are generically perpendicular (with downstream magnetic field quasi perpendicular to the shock normal, preventing particle from diffusing back upstream) and that particle isotropization upstream is no longer guaranteed (there being not sufficient time to growth turbulence on scales $\left.r_{\text {gyro,UHECR }}\right)$. The situation is relaxed for mildly relativistic shock speeds, and this has led to the recent proposal that UHECR particle acceleration may instead occur at multiple (mildly relativistic) shocks in the back-flows of radio galaxies [48]. Multiple shocks would provide multiple opportunities for acceleration, and also lead to harder spectra. The proposal is motivated by two- and three-dimensional hydrodynamical simulations of light (density contrast $\left.\rho_{j} / \rho_{0} \sim 10^{-5}-10^{-4}\right)$, high-power $\left(\sim 10^{45} \mathrm{erg} / \mathrm{sec}\right)$ jets in cluster environments, in which strong back-flows are seen (cf. also [49, 50]), that can be supersonic and exhibit related compression structures, cf. Fig. 7. Analysis of the simulation data suggests that about $10 \%$ of the particles pass through a shock of Mach number $M>3$, while $\sim 5 \%$ pass through multiple shocks. Inferred shock speeds are of the order of $u_{s} \sim 0.2 \mathrm{c}$, with estimated sizes $R \sim 2$ kpc. Assuming a reference magnetic field strength of $B \sim 100 \mu \mathrm{G}$, maximum $\mathrm{CR}$ energies of 


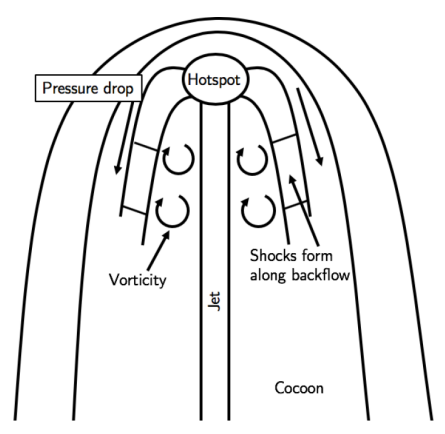

Figure 7: Cartoon of the considered scenario, where multiple (mildly relativistic) shocks in strong backflows provide sites for efficient CR acceleration to UHE energies. From ref. [18].

$E_{\max } \simeq 4 \times 10^{19} \mathrm{Z}\left(B / 10^{-4} \mathrm{G}\right)(R / 2 \mathrm{kpc}) \mathrm{eV}$, see eq. (3.2), could thus be achievable. While radio galaxies can in principle be powerful enough to satisfy the minimum power requirement $L_{m}$, eq. (3.4), this is currently barely the case within the GZK horizon. As CR are likely to escape slowly from a source, however, the past activity of a source would be relevant (see above). There are good reasons, for example, to consider an enhanced activity in the past driving the giant lobe evolution in Cen A. In the present context these lobes may then represent reservoirs of UHECRs.

\section{Conclusion and Perspectives}

Current correlation studies provide some indications that both starburst galaxies and AGN could play a significant role in the production of UHECRs. From a physical (acceleration) point of view, starburst galaxies are less promising, while radio galaxies appear capable of satisfying the relevant efficiency requirements. In particular, Fermi-type particle acceleration at trans-relativistic (internal) shocks and/or in shearing, relativistic jet flows could facilitate the production of UHECRs in jetted AGNs. This could be compatible with recent findings suggesting an increased $(\sim 4 \sigma)$ excess of UHECR $(E>39 \mathrm{eV})$ events around Cen A. Further experimental constraints on the chemical composition as well as more complex (and physically motivated) correlation studies will help to eventually conclude about the astrophysical sources of UHECRs.

Acknowledgement: I am grateful to the organisers for the invitation to a stimulating conference. I thank James Matthews and Kohta Murase for the allowance to use figures from their papers. Funding by a DFG Heisenberg Fellowship RI 1187/6-1 is kindly acknowledged.

\section{References}

[1] Pierre Auger Collaboration, A. Aab, P. Abreu, M. Aglietta, I. A. Samarai, I. F. M. Albuquerque et al., Observation of a large-scale anisotropy in the arrival directions of cosmic rays above $8 \times 10^{18} \mathrm{eV}$, Science 357 (2017) 1266 [1709.07321].

[2] A. Castellina, Highlights from the Pierre Auger Observatory, in 36th International Cosmic Ray Conference (ICRC2019), vol. 36 of International Cosmic Ray Conference, p. 4, Jul, 2019, 1909.10791. 
[3] S. Ogio, Highlights from the Telescope Array, in 36th International Cosmic Ray Conference (ICRC2019), vol. 36 of International Cosmic Ray Conference, p. 13, Jul, 2019.

[4] M. Kachelriess and D. V. Semikoz, Cosmic Ray Models, Progress in Particle and Nuclear Physics (2019) arXiv:1904.08160 [1904.08160].

[5] L. A. Anchordoqui, Ultra-high-energy cosmic rays, Physics Reports 801 (2019) 1 [1807.09645].

[6] D. Ivanov, Pierre Auger Collaboration and Telescope Array Collaboration, Report of the Telescope Array - Pierre Auger Observatory Working Group on Energy Spectrum, in 35th International Cosmic Ray Conference (ICRC2017), vol. 301 of International Cosmic Ray Conference, p. 498, Jan, 2017.

[7] K. Murase and M. Fukugita, Energetics of high-energy cosmic radiations, Phys Rev D 99 (2019) $063012[1806.04194]$.

[8] R. Alves Batista, J. Biteau, M. Bustamante, K. Dolag, R. Engel, K. Fang et al., Open questions in cosmic-ray research at ultrahigh energies, Frontiers in Astronomy and Space Sciences 6 (2019) 23 [1903.06714].

[9] J. Matthews and Telescope Array Collaboration, Highlights from the Telescope Array Experiment, in 35th International Cosmic Ray Conference (ICRC2017), vol. 301 of International Cosmic Ray Conference, p. 1096, Jan, 2017.

[10] K. Kawata, A. di Matteo, T. Fujii, D. Ivanov, C. C. H. Jui, J. P. Lundquist et al., TA Anisotropy Summary, in European Physical Journal Web of Conferences, vol. 210 of European Physical Journal Web of Conferences, p. 01004, Oct, 2019, DOI.

[11] M. Lemoine and E. Waxman, Anisotropy vs chemical composition at ultra-high energies, JCAP 2009 (2009) 009 [0907.1354].

[12] R.-Y. Liu, A. M. Taylor, M. Lemoine, X.-Y. Wang and E. Waxman, Constraints on the Source of Ultra-high-energy Cosmic Rays Using Anisotropy versus Chemical Composition, ApJ 776 (2013) 88 [1308.5699].

[13] M. Lemoine, On Ultra-High Rigidity Cosmic Rays, in Ultra-High Energy Cosmic Rays (UHECR2016), p. 011004, Jan, 2018, DOI.

[14] A. Aab, P. Abreu, M. Aglietta, I. F. M. Albuquerque, I. Allekotte, A. Almela et al., An Indication of Anisotropy in Arrival Directions of Ultra-high-energy Cosmic Rays through Comparison to the Flux Pattern of Extragalactic Gamma-Ray Sources, ApJL 853 (2018) L29 [1801 . 06160 ].

[15] L. Caccianiga, Anisotropies of the Highest Energy Cosmic-ray Events Recorded by the Pierre Auger Observatory in 15 years of Operation, in 36th International Cosmic Ray Conference (ICRC2019), vol. 36 of International Cosmic Ray Conference, p. 206, Jul, 2019.

[16] R. U. Abbasi, M. Abe, T. Abu-Zayyad, M. Allen, R. Azuma, E. Barcikowski et al., Testing a Reported Correlation between Arrival Directions of Ultra-high-energy Cosmic Rays and a Flux Pattern from nearby Starburst Galaxies using Telescope Array Data, ApJL 867 (2018) L27 [1 809.01573 ].

[17] J. H. Matthews, A. R. Bell, K. M. Blundell and A. T. Araudo, Fornax A, Centaurus A, and other radio galaxies as sources of ultrahigh energy cosmic rays, MNRAS 479 (2018) L76 [1805 . 01902 ].

[18] J. H. Matthews, A. R. Bell, A. T. Araudo and K. M. Blundell, Cosmic ray acceleration to ultrahigh energy in radio galaxies, in European Physical Journal Web of Conferences, vol. 210 of European Physical Journal Web of Conferences, p. 04002, Oct, 2019, 1902 .10382, DOI.

[19] B. Eichmann, J. P. Rachen, L. Merten, A. van Vliet and J. Becker Tjus, Ultra-high-energy cosmic rays from radio galaxies, JCAP 2018 (2018) 036 [1701. 06792]. 
[20] O. Kobzar, B. Hnatyk, V. Marchenko and O. Sushchov, Search for ultra high-energy cosmic rays from radiogalaxy Virgo A, MNRAS 484 (2019) 1790 [1810 . 10294].

[21] N. Fraija, M. Araya, A. Galván-Gámez and J. A. de Diego, Analysis of Fermi-LAT observations, UHECRs and neutrinos from the radio galaxy Centaurus B, JCAP 2019 (2019) 023 [1811.01108].

[22] A. M. Hillas, The Origin of Ultra-High-Energy Cosmic Rays, ARA\&A 22 (1984) 425.

[23] C. A. Norman, D. B. Melrose and A. Achterberg, The Origin of Cosmic Rays above $10^{18.5} \mathrm{eV}$, ApJ 454 (1995) 60.

[24] R. D. Blandford, Acceleration of Ultra High Energy Cosmic Rays, Physica Scripta Volume T 85 (2000) 191 [astro-ph/9906026].

[25] F. A. Aharonian, A. A. Belyanin, E. V. Derishev, V. V. Kocharovsky and V. V. Kocharovsky, Constraints on the extremely high-energy cosmic ray accelerators from classical electrodynamics, Phys Rev D 66 (2002) 023005 [astro-ph/0202229].

[26] G. Katsoulakos and F. M. Rieger, Magnetospheric Gamma-Ray Emission in Active Galactic Nuclei, ApJ 852 (2018) 112 [1712.04203].

[27] G. E. Romero, A. L. Müller and M. Roth, Particle acceleration in the superwinds of starburst galaxies, A\&A 616 (2018) A57 [1801.06483].

[28] A. Levinson, Particle Acceleration and Curvature TeV Emission by Rotating, Supermassive Black Holes, Phys Rev Lett 85 (2000) 912.

[29] A. Y. Neronov, D. V. Semikoz and I. I. Tkachev, Ultra-high energy cosmic ray production in the polar cap regions of black hole magnetospheres, New Journal of Physics 11 (2009) 065015 [0 712 . 1737].

[30] F. M. Rieger, Nonthermal Processes in Black Hole-Jet Magnetospheres, International Journal of Modern Physics D 20 (2011) 1547 [1107.2119].

[31] K. Ptitsyna and A. Neronov, Particle acceleration in the vacuum gaps in black hole magnetospheres, A\&A 593 (2016) A8 [1510.04023].

[32] R. J. Moncada, R. A. Colon, J. J. Guerra, M. J. O’Dowd and L. A. Anchordoqui, Ultrahigh energy cosmic ray nuclei from remnants of dead quasars, Journal of High Energy Astrophysics 13 (2017) 32 [1702.00053].

[33] F. Rieger and A. Levinson, Radio Galaxies at VHE Energies, Galaxies 6 (2018) 116 [1810 . 05409 ].

[34] G. Pedaletti, S. J. Wagner and F. M. Rieger, Very High Energy $\gamma$-ray Emission from Passive Supermassive Black Holes: Constraints for NGC 1399, ApJ 738 (2011) 142 [1107. 0910].

[35] F. M. Rieger, An Introduction to Particle Acceleration in Shearing Flows, Galaxies 7 (2019) 3 [1909.07237].

[36] M. Ostrowski, Acceleration of ultra-high energy cosmic ray particles in relativistic jets in extragalactic radio sources, A\&A 335 (1998) 134 [astro-ph/9803299].

[37] M. Ostrowski, On possible 'cosmic ray cocoons' of relativistic jets, MNRAS 312 (2000) 579 [astro-ph/9910491].

[38] D. Caprioli, “Espresso” Acceleration of Ultra-high-energy Cosmic Rays, ApJL 811 (2015) L38 [1505.06739].

[39] S. S. Kimura, K. Murase and B. T. Zhang, Ultrahigh-energy cosmic-ray nuclei from black hole jets: Recycling galactic cosmic rays through shear acceleration, Phys Rev D 97 (2018) 023026 [1705.05027]. 
[40] F. M. Rieger and P. Duffy, Shear Acceleration in Relativistic Astrophysical Jets, ApJ 617 (2004) 155 [astro-ph/0410269].

[41] F. M. Rieger and P. Duffy, Shear Acceleration in Expanding Flows, ApJ 833 (2016) 34 [1611.04342].

[42] R.-Y. Liu, F. M. Rieger and F. A. Aharonian, Particle Acceleration in Mildly Relativistic Shearing Flows: The Interplay of Systematic and Stochastic Effects, and the Origin of the Extended High-energy Emission in AGN Jets, ApJ 842 (2017) 39 [1706.01054].

[43] G. M. Webb, A. F. Barghouty, Q. Hu and J. A. le Roux, Particle Acceleration Due to Cosmic-ray Viscosity and Fluid Shear in Astrophysical Jets, ApJ 855 (2018) 31.

[44] G. M. Webb, S. Al-Nussirat, P. Mostafavi, A. F. Barghouty, G. Li, J. A. le Roux et al., Particle Acceleration by Cosmic Ray Viscosity in Radio-jet Shear Flows, ApJ 881 (2019) 123.

[45] M. Lemoine and G. Pelletier, On electromagnetic instabilities at ultra-relativistic shock waves, MNRAS 402 (2010) 321 [0904.2657].

[46] L. Sironi and A. Spitkovsky, Particle Acceleration in Relativistic Magnetized Collisionless Electron-Ion Shocks, ApJ 726 (2011) 75 [1009.0024].

[47] A. R. Bell, A. T. Araudo, J. H. Matthews and K. M. Blundell, Cosmic-ray acceleration by relativistic shocks: limits and estimates, MNRAS 473 (2018) 2364 [1709.07793].

[48] J. H. Matthews, A. R. Bell, K. M. Blundell and A. T. Araudo, Ultrahigh energy cosmic rays from shocks in the lobes of powerful radio galaxies, MNRAS 482 (2019) 4303 [1810 . 12350].

[49] M. Perucho and J. M. Martí, A numerical simulation of the evolution and fate of a Fanaroff-Riley type I jet. The case of 3C 31, MNRAS 382 (2007) 526 [0709.1784].

[50] M. Perucho, J.-M. Martí and V. Quilis, Long-term FRII jet evolution: clues from three-dimensional simulations, MNRAS 482 (2019) 3718 [1810.10968]. 